\title{
Multicomponent dark matter
}

\author{
Kathryn M. Zurek \\ Particle Astrophysics Center, Fermi National Accelerator Laboratory, Batavia, Illinois 60510 \\ and Department of Physics, USAUniversity of Michigan, Ann Arbor, Michigan 48109, USA
}

(Received 10 January 2009; published 5 June 2009)

\begin{abstract}
We explore multicomponent dark matter models where the dark sector consists of multiple stable states with different mass scales, and dark forces coupling these states further enrich the dynamics. The multicomponent nature of the dark matter naturally arises in supersymmetric models, where both $R$ parity and an additional symmetry, such as a $Z_{2}$, is preserved. We focus on a particular model where the heavier component of dark matter carries lepton number and annihilates mostly to leptons. The heavier component, which is essentially a sterile neutrino, naturally explains the PAMELA and synchrotron signals, without an excess in antiprotons, which typically mars other models of weak scale dark matter. The lighter component, which may have a mass from a GeV to a TeV, may explain the DAMA signal, and may be visible in low threshold runs of CDMS and XENON, which search for light dark matter.
\end{abstract}

DOI: 10.1103/PhysRevD.79.115002

PACS numbers: $95.35 .+\mathrm{d}, 12.60 . \mathrm{Jv}$

There have been many tantalizing signals that may be evidence for particle dark matter. Most recently, the PAMELA experiment has reported a cosmic ray positron excess of positrons with energy in the $10-100 \mathrm{GeV}$ range [1], which is consistent with annihilating dark matter [2], confirming the excess observed by the HEAT [3] and AMS [4] experiments. The ATIC and PPB-BETS balloon experiments have likewise observed an excess, consistent with the PAMELA, HEAT, and AMS results. ATIC and PPBBETS suggest a dark matter particle annihilating to leptons with mass in the 500-800 GeV range [5]; the other observations are consistent with dark matter mass in this range. The recent Fermi Large Area Telescope results [6] suggest, however, that the ATIC excess may be instrumental in origin. If this is the case, the annihilating dark matter particle may be much lighter, with mass in the $\sim 100-200 \mathrm{GeV}$ range to explain the PAMELA excess only [7]. To explain both the PAMELA and Fermi excesses by dark matter, however, its mass should be even higher, in the $\sim 1 \mathrm{TeV}$ range [8]. In addition, there is the observation of the synchrotron radiation toward the galactic center, the so-called "WMAP haze," which is indicative of dark matter annihilating to electrons that emit photons in the galactic magnetic field [9]. Indeed, an annihilation cross section to $e^{+} e^{-}$, which produces the WMAP haze is roughly the right size (up to a boost factor) to produce the AMS, HEAT, ATIC, PPB-BETS, and PAMELA excesses. The size of these signals is also roughly consistent with the freeze-out annihilation cross section predicted for a thermal relic weakly interacting massive particle (WIMP). In direct detection, the DAMA experiment has reported an $8.2 \sigma$ significance modulation in the rate of recoils in their experiment [10]. The phase and amplitude of their signal is consistent with a light elastically scattering WIMP with mass in the $\sim 3-10 \mathrm{GeV}$ range [11] (though see [12] for a discussion of the effect of the lowest DAMA recoil bin on the fit in this window).
While these signals are intriguing, detailed explanations of these signals in terms of standard models of WIMP dark matter, such as supersymmetry, may be challenging. One difficulty in explaining the AMS, HEAT, PAMELA, ATIC, PPB-BETS, and haze excesses is that the dark matter must have a large annihilation cross section to leptons and a small annihilation cross section to hadrons, since the data shows a positron excess but no excess of antiprotons $[1,7,13]$. This is challenging for two reasons. First, hadrons carry an enhancement in the annihilation cross section that goes like $N_{c}$, the number of colors; hence, in many models, annihilation to colored particles is the preferred mode. Secondly, when the dark matter particle is Majorana, as in supersymmetry (SUSY) models, there is a chiral suppression that disfavors annihilation to light modes. In SUSY, annihilation to $\bar{b} b, \tau^{+} \tau^{-}$, and $W^{+} W^{-}$is preferred; it has been shown that an annihilation cross section big enough to produce the positron excess through this mode will produce too many antiprotons through the hadronic decays of these states (see e.g. $[13,14]$ for the case of $W^{+} W^{-}$).

In this paper we develop models that naturally overcome this challenge, where the dark matter effectively carries lepton number, and hence annihilation to leptons is the only mode allowed. We also show that within this class of models, the dark matter may in fact also quite naturally be multicomponent. A heavier component explains the PAMELA (and perhaps Fermi) and synchrotron excesses, while the lighter component, residing in the hidden sector, may have a much lower mass, and may explain the DAMA signal. These low mass states may be reachable with low threshold analyses currently being planned by the CDMS and XENON experiments [15].

The addition of these low mass hidden sectors with multicomponent dark matter naturally suggests rich dynamics in the hidden sector. In many cases, there are new forces, both scalar and vector, which give rise to novel 
phenomenology, and in many ways, the rich dynamics of these low mass hidden sector dark matter models is motivated by the hidden valley [16]. The components of the model we discuss here, with multiple dark forces and low mass dark matter states coupled to the standard model (SM) through kinetic mixing or $\mathrm{TeV}$ mass states, resemble features of the low mass hidden dark matter models constructed in [17-19]. Because multiple forces may reside in the hidden sector, these models may also provide a natural context for solving a second challenge for a model of dark matter (DM) explaining the positron excesses. That is, there must be a boost in the annihilation of the dark matter in the halo today relative to the cross section required at thermal freeze-out $\langle\sigma v\rangle \sim 3 \times 10^{-26} \mathrm{~cm}^{3} / \mathrm{s}$. For dark matter in the $500-1000 \mathrm{GeV}$ range, the boost is typically quite large, $\sim 100-1000$, for direct annihilation to $e^{+} e^{-}$ [5,7]. A smaller, though still significant, boost is required for lighter DM in the $\sim 100 \mathrm{GeV}$ range [7]. The boost factor may come from a large overdensity in the dark matter locally in the galaxy, though simulations suggest that a boost factor much larger than $\sim 5$ is difficult to produce. A boost factor may instead imply that the size of the dark matter annihilation cross section in the halo today is larger than the annihilation at thermal freeze-out. A possible source of the needed enhancement of the cross section today is the so-called Sommerfeld effect $[13,20]$. This effect gives rise to an enhancement of the annihilation cross section at low velocity $v$, so that the annihilation cross section for particles locally in our halo $\left(v \sim 10^{-3}\right)$ is enhanced with respect to the freeze-out cross section $(v \sim$ 0.3 ). One of the additional dark forces may provide for such an enhancement. (See [21] for a model where late decay of a meta-stable state produces the needed boost.)

Models with such large boosted annihilation cross sections potentially run into phenomenological constraints. It was shown in [13] that if the dark matter density profile in the inner $200 \mathrm{pc}$ is much steeper than an isothermal profile, gamma ray constraints from HESS in the galactic center and galactic ridge require $B\langle\sigma v\rangle \lesssim 10^{-24} \mathrm{~cm}^{3} / \mathrm{s}$, where $B$ is the astrophysical boost factor, for dark matter in the several hundred $\mathrm{GeV}$ mass window. This constraint is problematic for the window preferred by ATIC and Fermi for larger dark matter masses. For smaller dark matter masses consistent with the PAMELA signal the constraint is less significant due to the smaller annihilation crosssections required. The constraint can be alleviated, however, even for dark matter in the several hundred $\mathrm{GeV}$ range. For example, the density profile may be flatter than a Navarro-Frenk-White or Einasto profile in the inner 200 pc. Or the astrophysical boost factor may be smaller in the galactic center than it is at the solar radius, where the positrons originate. This is expected where tidal disruption of dense objects in the galactic center occurs. Thus, while $B\langle\sigma v\rangle \lesssim 10^{-24} \mathrm{~cm}^{3} / \mathrm{s}$ at the galactic center, $B\langle\sigma v\rangle$ may be much larger locally to produce the large positron ex- cesses. A second less stringent constraint on these Sommerfeld boosted cross sections is derived from big bang nucleosynthesis [22], $\langle\sigma v\rangle \lesssim 7 \times 10^{-24} \mathrm{~cm}^{3} / \mathrm{s}$ for annihilation to electrons and $\langle\sigma v\rangle \lesssim 2 \times 10^{-23} \mathrm{~cm}^{3} / \mathrm{s}$ for annihilation to muons and taus. These constraints can be satisfied even for moderately large dark matter masses in the class of models we consider with astrophysical boost factors that are on the order of a few.

Although dark matter with multiple components could potentially be quite complicated, in this paper we propose that the dark sectors take on a simple basic structure. (See [23] for another model of dark matter with more than one stable state.) To the SM sector, we add an " $X$ sector." The $X$ sector contains generally high mass states, in the 100's of $\mathrm{GeV}-1 \mathrm{TeV}$ range, which communicate to the SM through $\mathcal{O}(1)$ operators that carry lepton number. Thus, the lightest state in this sector annihilates primarily to charged lepton (or unobserved neutrino) pairs, producing the observed PAMELA and synchrotron excesses, without any excess in antiprotons. The dark matter is essentially a sterile neutrino, which is stable by virtue of a $Z_{2}$ symmetry.

When such a model is supersymmetrized, there is now a second stable state by a $R$ parity. To the $X$ sector, we may add a hidden dark matter (hDM) sector. The hDM sector does two things. First, the hDM sector makes the minimal supersymmetric standard model (MSSM) lightest supersymmetric partner (LSP) unstable to decay to $\mathrm{hDM}$ states with the same $R$-symmetry charge. Thus, the lightest $R$-symmetry odd state may in fact be much lighter than the weak scale. This was pointed out in [24] for hidden valleys and applied to dark matter in the context of a supersymmetric $\mathrm{MeV}$ hidden sector in [19]. Here, we are interested in the case where that state has a mass in the 1$10 \mathrm{GeV}$ range, and explains the DAMA signal. Second, the $\mathrm{hDM}$ sector provides a means for breaking the symmetry of the new dark forces and giving masses to the gauged mediators. In some cases, these forces may give rise to a Sommerfeld enhancement. The mass of those dark forces should be in the sub-50 GeV range, since the Sommerfeld enhancement is effective for mediator masses $m_{M}$ satisfying $g_{D}^{2} M_{X} /(4 \pi) \gtrsim m_{M}$, where $g_{D}$ is the coupling of the dark force to the dark matter $X$. Thus, for $g_{D} \sim 0.1-1$ we see that the $1-10 \mathrm{GeV}$ range is motivated both for mediators of the Sommerfeld effect and for a dark matter candidate to explain the DAMA signal.

Such light scalar or gauged mediators are natural in the presence of hidden sectors, as shown in [19] in the context of $\mathrm{MeV}$ dark matter. On the basis of naturalness considerations, one expects scalar forces (or massive gauged particles, which get their masses from such scalars) to be at the weak scale. However, such light scalars can be natural if the hidden sector is shielded from MSSM SUSY breaking (which tends to push the mass of the force mediators to the weak scale) by a weak coupling to the MSSM sector. We will consider the case where the weak 
coupling is either a mixing angle $\theta$ between the dark force $U(1)_{D}$ and hypercharge $U(1)_{Y}$, or a small coupling $\lambda_{D}$ of a visible sector singlet scalar with the hDM sector. These weak couplings set the mass scale $m_{D}$ in the hDM sector to be $m_{D} \sim \theta m_{\mathrm{SUSY}}$, or $m_{D} \sim \lambda_{D} m_{\mathrm{SUSY}}$ (up to loop factors), where $m_{\text {SUSY }} \sim 0.1-1 \mathrm{TeV}$ are the MSSM SUSY breaking masses, and $m_{D}$ is the typical scale for the dark forces and the DM in the hidden sector. Since the kinetic mixing between the two sectors may typically be a loop factor $\theta \sim$ $10^{-2}$, or a somewhat small coupling $\lambda_{D} \sim 10^{-1-2}$, the low mass $1-10 \mathrm{GeV}$ scale is further motivated. While such a mechanism was introduced in the context of $\mathrm{MeV}$ dark matter for smaller mixings $\theta \sim 10^{-5}$, it was shown to be quite general for higher mass hidden sectors in the $0.1-$ $100 \mathrm{GeV}$ range [25,26].

We now turn to constructing the $X$ model explicitly. To the MSSM we add

$$
\Delta W=y_{i}^{\prime} L_{i} H^{\prime} \bar{X}+\lambda_{X} S_{X} \bar{X} X+\kappa_{X} S_{X}^{3}
$$

where $H^{\prime}$ is an electroweak doublet. There is a $Z_{2}$ symmetry under which $H^{\prime}$ and $X$ are odd, and also an $R$ symmetry. If a component of $X$ is the lightest $Z_{2}$ odd particle, it is a stable dark matter candidate, and it effectively carries lepton number, explaining why it annihilates predominantly to leptons (for another leptophilic model see [27]) through $t$-channel $H^{\prime}$ exchange. The mass of such a dark matter state is in the 100's of GeV range, and it must be fermion to get an $s$-wave annihilation cross section (which is unsuppressed at low velocities in the halo today). The annihilation cross section is $\langle\sigma v\rangle=y_{i}^{\prime 4} m_{X}^{2} /\left(16 \pi m_{H^{\prime}}^{4}\right)$, which must be $\langle\sigma v\rangle \simeq 3 \times 10^{-26} \mathrm{~cm}^{3} / \mathrm{s}$ in order to be consistent with the observed relic abundance. Thus, for $m_{X} \approx 700 \mathrm{GeV}$, we find $y_{i}^{\prime} \lesssim 0.6$. The scalar component $\tilde{X}$ annihilating through $t$-channel Higgsino $\tilde{H}^{\prime}$ exchange to $e^{+} e^{-}$, on the other hand, gives a $p$-wave suppressed annihilation. Thus, to have a viable model, $\tilde{X}$ must be heavier than $X$, and rapidly decay to the $X$ fermion plus the lightest $R$-symmetry odd state, which will reside in the hDM sector. With this annihilation cross section to electronpositron pairs, the rate is a factor $\sim 100-1000$ below what is required to reproduce the ATIC and PAMELA signals together, and a factor $\sim 10$ below what is required to produce the PAMELA signal alone. The required boost from a Sommerfeld enhancement may be mediated by a singlet scalar $S_{X}$, which generates the mass for $X$, $\lambda_{X}\left\langle S_{X}\right\rangle=m_{X}$. This enhancement is relevant if $\lambda_{X}^{2} /(4 \pi) m_{X} \gtrsim m_{S_{X}}$ is satisfied [20]. Since $\left\langle S_{X}\right\rangle=\frac{m_{S_{X}}}{3 \kappa_{X}}$, we find that the Sommerfeld condition is satisfied if $\frac{\lambda_{X}^{3}}{12 \pi} \geq$ $\kappa_{X}$, which is fulfilled for $\lambda_{X} \simeq 1$ and a relatively small $\kappa_{X}$. This singlet $S_{X}$ must have a relatively small mixing angle with the Higgs in order not to violate direct detection bounds for the DM candidate $X$ (though it may be possible that this scalar is that of the next to minimal supersymmetric standard model, see [28] for a possible model). We will see next that one of the scalars residing in the hDM sector may also quite naturally mediate the boost.

To this point, we have two stable states: the DM fermion $X$ stable by the $Z_{2}$ and the LSP (either the scalar $\tilde{X}$ or an MSSM superpartner). With the addition of a supersymmetrized low mass hidden sector, the LSP becomes unstable to decay to the hidden sector, so that the LSP mass may be much lighter than the weak scale. For the purposes of this toy model, we consider the minimal hDM superpotential

$$
W_{h}=\lambda_{D} S_{D} \bar{D} D+\kappa_{D} S_{D}^{3} .
$$

This hidden toy model is fashioned after that discussed in [19], and is to be added to the $X$-sector superpotential, Eq. (1). Here, $S_{D}$ is a dark singlet field, and the dark Higgses $\bar{D}, D$ may be charged under a new hidden gauge group $U(1)_{D}$, which is a dark force. $U(1)_{D}$ mixes with hypercharge through the kinetic term $\theta F_{D}^{\mu \nu} F_{\mu \nu}$.

The lightest state in this hDM sector may be a candidate to explain the DAMA signal, if its mass is in the $1-10 \mathrm{GeV}$ range. This mass may naturally be induced radiatively from two sources. First, kinetic mixing between hypercharge and $U(1)_{D}$ is $\theta \sim 10^{-2}-10^{-3}$, as expected when the mixing is induced by a loop of heavy particles [29]. This kinetic mixing introduces SUSY breaking into the hidden sector by a two loop gauge mediation diagram, with messengers in the loop, as in [19]. We term this mechanism for SUSY breaking in the hidden sector "little gauge mediation." The size of the radiatively induced $D, \bar{D}$ masses is $m_{D, \text { rad }}^{2}=3 / 5 g_{D}^{2} g_{Y}^{2} \theta^{2} m_{\text {SUSY }}^{2}$, where $m_{\text {SUSY }}=$ $\left\langle F_{\text {mess }}\right\rangle /\left(16 \pi^{2} M_{\text {mess }}\right)$ is the SUSY breaking mass in the messenger sector, $g_{D}$ is the gauge coupling of $U(1)_{D}$, and $g_{Y}$ the hypercharge gauge coupling. With $\theta \sim 10^{-2}-$ $10^{-3}$, and $\mathcal{O}(1)$ couplings, we can see that the $\mathrm{GeV}$ mass scale is naturally generated in the hidden sector. In order to break $U(1)_{D}$, this mass squared must be negative. One loop graphs with the scalar $S_{D}$ in the loop may easily induce such a negative mass squared $m_{D, \text { rad }}^{2} \simeq-\frac{4 \lambda_{D}^{4} m_{S_{D}}^{2}}{16 \pi^{2}} \log \left(\frac{\Lambda^{2}}{m_{\text {SUSY }}^{2}}\right)$, where $\Lambda$ is the scale where the soft masses are generated, and $m_{S_{D}}^{2}$ is the soft SUSY breaking mass of $S_{D}$ (we assume that the singlet receives a moderate SUSY breaking mass in the 10 to 100's of GeV range through a coupling to the SUSY breaking messenger fields). For $\lambda_{D} \approx 10^{-1}-1$, soft masses for $D$ in the few $\mathrm{GeV}$ range result that are negative, even with the contribution from little gauge mediation included.

With $\left\langle S_{D}\right\rangle=0$ and $\langle D, \bar{D}\rangle \neq 0$, we review the spectrum briefly. With $\mathcal{O}\left(10^{-1-2}\right)$ gauge coupling $g_{D}$ and Yukawa term $\lambda_{D}$, all masses in the hidden sector are $\mathcal{O}(\mathrm{GeV})$. The $U(1)_{D}$ symmetry is broken by $\langle D, \bar{D}\rangle$, and the gauge boson acquires a mass. We have scalar mass eigenstates $m_{D_{1}}^{2}=$ $-\frac{4 g_{D}^{2}-2 \lambda_{D}^{2}}{\lambda_{D}^{2}} m_{D, \text { rad }}^{2}, m_{D_{2}}^{2}=-2 m_{D, \text { rad }}^{2}$, and $m_{U_{D}}^{2}=4 g_{D}^{2}\langle D\rangle^{2}$ from the breaking of the $U(1)_{D}$ with $\langle D, \bar{D}\rangle^{2}=$ $-m_{D, \text { rad }}^{2} / \lambda_{D}^{2}$. The fermion masses arise through $\tilde{D}, \tilde{\bar{D}}$, 
$\tilde{U}_{D}, \tilde{S}_{D}$ mixing, two with masses $2 g_{D}\langle D\rangle\left(\right.$ a $\tilde{U}_{D}$ gaugino- $\tilde{D}$ Higgsino mix) and two with masses $\sqrt{2} \lambda_{D}\langle D\rangle$ (a $\tilde{S}_{D}$ singlino- $\tilde{D}$ Higgsino mix). We assume $g_{D} \gtrsim \lambda_{D}$ so that the fermions with mass $\sqrt{2} \lambda_{D}\langle D\rangle$ are stable dark matter candidates, provided they are lighter than the gravitino.

Now, we can see that such a sector can plausibly give rise to a signal in DAMA in the elastically scattering WIMP window. We take the $\tilde{D}-\tilde{S}_{D}$ fermions to be the dark matter with mass $m_{\mathrm{hDM}}$ in the $3-10 \mathrm{GeV}$ range. The DM may annihilate to the axion associated with the angular components of $D, \bar{D}$, which is light, as in the next to minimal supersymmetric standard model. The annihilation cross section of the hidden dark matter to these axions is

$$
\sigma_{\mathrm{ann}} \simeq \frac{\lambda_{D}^{4}}{16 \pi} \frac{1}{m_{\mathrm{hDM}}^{2}} \sim 10^{-35} \mathrm{~cm}^{2}\left(\frac{\lambda_{D}}{0.1}\right)^{2}\left(\frac{8 \mathrm{GeV}}{m_{\mathrm{hDM}}}\right)^{2} .
$$

This cross section is of the order $\sim 10^{-36} \mathrm{~cm}^{2}$ necessary to produce the correct relic density (this candidate need not be all the dark matter). The direct detection cross section by exchanging a $U_{D}$ gauge boson is

$$
\sigma_{\mathrm{SI}} \simeq \frac{g_{D}^{2} g_{Y}^{2} \theta^{2}}{\pi} \frac{m_{r}^{2}}{m_{U_{D}}^{4}} \sim 10^{-40} \mathrm{~cm}^{2}\left(\frac{g_{D} g_{Y} \theta}{10^{-4}}\right)^{2}\left(\frac{8 \mathrm{GeV}}{m_{U_{D}}}\right)^{4},
$$

where $m_{r}$ is the reduced mass of the nucleon-DM system. We see that a hidden sector, which simultaneously generates natural $\mathrm{GeV}$ mediators and $\mathrm{GeV}$ scale dark matter candidates, produces a direct detection cross section in a range to be the explanation for the DAMA signal.

In addition, if the singlet $S_{D}$ couples to the visible Higgs through a term in the superpotential $\zeta S_{D} H_{u} H_{d}$, this provides an additional channel for direct detection. The size of the scattering cross section is

$$
\begin{aligned}
\sigma_{n} \simeq & \frac{m_{r}^{2}}{2 \pi} N_{n}^{2}\left(\frac{\lambda_{D} \zeta v_{u}\langle D\rangle}{m_{h^{0}}^{2}}\right)^{2} \frac{1}{m_{D_{1}}^{4}} \\
\simeq & 2 \times 10^{-41} \mathrm{~cm}^{2}\left(\frac{N_{n}}{0.1}\right)^{2}\left(\frac{\lambda_{D}}{0.1}\right)^{2}\left(\frac{\zeta}{10^{-2}}\right)^{2}\left(\frac{\langle D\rangle}{20 \mathrm{GeV}}\right)^{2} \\
& \times\left(\frac{100 \mathrm{GeV}}{m_{h^{0}}}\right)^{4}\left(\frac{10 \mathrm{GeV}}{m_{D_{1}}}\right)^{4},
\end{aligned}
$$

where $N_{n}$ comes from the effective coupling of the exchanged scalar to the target nucleus, and $h^{0}$ is the MSSM Higgs. We see again that this mechanism results in a scattering cross section is in the $10^{-41}-10^{-39} \mathrm{~cm}^{2}$ window for explaining the DAMA signal with light WIMPs (if the light state only composes a fraction of the DM, scattering cross sections should be correspondingly larger). Alternatively, if the DAMA signal turns out not to be from DM scattering, it is easy to evade direct detection bounds by lowering the mixing $\zeta$ or correspondingly raising the mass of the mediators; these lower mass WIMPs may still be in reach of the low threshold runs of CDMS [15] and XENON.

The general conclusion here is that such hidden sectors with $\mathrm{GeV}$ mass dark matter particles and dark forces of $\mathrm{GeV}$ mass mediators arise naturally in a framework where the hidden sector communicates to the SM through kinetic mixing of dark force with hypercharge, or through mixing of a singlet scalar with both the hidden and visible sectors. The mixing simultaneously provides motivation for observation of these states by direct detection experiments. These light gauged or scalar mediators may in fact mediate the Sommerfeld enhancement as well, if $X$ is charged under the $U(1)_{D}$, or if $S_{D}$ also couples to $X$ in addition to $D$.

We have discussed multicomponent dark matter models in which the dark sector is more complex than a single weakly interacting field. In many cases, these models give rise to additional dark forces that enrich the dark matter dynamics. Phenomenologically, the focus of this paper has been on explanations of the PAMELA, ATIC, PPB-BETS, HEAT, AMS, and DAMA excesses. In the models discussed here, the dark matter candidate that explains the positron excess carries lepton number; it is stable by an additional $Z_{2}$ symmetry. We showed that in supersymmetric models of this type, there are naturally two dark matter candidates - the lighter candidate may explain the DAMA signal, and may be observable by low threshold runs of CDMS, XENON. We also showed how dark forces that arise in hidden sector dark matter models may naturally have their masses generated at the $\mathrm{GeV}$ scale, further motivating the low mass WIMP window as a wellmotivated scale for direct detection of dark matter. Dark matter dynamics and dark matter sectors may be rich. As multiple experiments with varied detection techniques probe the dark sector, we may discover a dark hidden world in lieu of a single weakly interacting particle.

This work has been supported by the U.S. Department of Energy, including Grant No. DE-FG02-95ER40896, and by NASA Grant No. NAG5-10842. We thank P. Fox, D. Hooper, P. Ouyang, F. Petriello, and E. Poppitz for discussions, and A. Strumia for a comment on the first version.
[1] O. Adriani et al., Nature (London) 458, 607 (2009); Phys. Rev. Lett. 102, 051101 (2009).

[2] A. J. Tylka, Phys. Rev. Lett. 63, 840 (1989); 63, 1658(E)
(1989); G. L. Kane, L. T. Wang, and J. D. Wells, Phys. Rev. D 65, 057701 (2002); E. A. Baltz, J. Edsjo, K. Freese, and P. Gondolo, Phys. Rev. D 65, 063511 (2002); G. L. Kane, 
L. T. Wang, and T. T. Wang, Phys. Lett. B 536, 263 (2002); D. Hooper, J.E. Taylor, and J. Silk, Phys. Rev. D 69, 103509 (2004).

[3] S. W. Barwick et al. (HEAT Collaboration), Astrophys. J. 482, L191 (1997); J. J. Beatty et al., Phys. Rev. Lett. 93, 241102 (2004).

[4] M. Aguilar et al. (AMS-01 Collaboration), Phys. Lett. B 646, 145 (2007).

[5] S. Torii et al., Nature (London) 456, 362 (2008); S. Torii, arXiv:0809.0760.

[6] A. A. Abdo et al. (Fermi LAT Collaboration), Phys. Rev. Lett. 102, 181101 (2009).

[7] I. Cholis, L. Goodenough, D. Hooper, M. Simet, and N. Weiner, arXiv:0809.1683.

[8] P. Meade, M. Papucci, A. Strumia, and T. Volansky, arXiv:0905.0480.

[9] D. P. Finkbeiner, Astrophys. J. 614, 186 (2004); G. Dobler and D. P. Finkbeiner, Astrophys. J. 680, 1222 (2008); D. Hooper, D. P. Finkbeiner, and G. Dobler, Phys. Rev. D 76, 083012 (2007).

[10] R. Bernabei et al. (DAMA Collaboration), Eur. Phys. J. C 56, 333 (2008).

[11] C. Savage, K. Freese, P. Gondolo, and D. Spolyar, arXiv:0901.2713; C. Savage, G. Gelmini, P. Gondolo, and K. Freese, J. Cosmol. Astropart. Phys. 04 (2009) 010; F. Petriello and K. M. Zurek, J. High Energy Phys. 09 (2008) 047; A. Bottino, F. Donato, N. Fornengo, and S. Scopel, Phys. Rev. D 78, 083520 (2008).

[12] S. Chang, A. Pierce, and N. Weiner, arXiv:0808.0196; M. Fairbairn and T. Schwetz, J. Cosmol. Astropart. Phys. 01 (2009) 037.
[13] M. Cirelli, M. Kadastik, M. Raidal, and A. Strumia, J. Cosmol. Astropart. Phys. 03 (2009) 009.

[14] F. Donato, D. Maurin, P. Brun, T. Delahaye, and P. Salati, Phys. Rev. Lett. 102, 071301 (2009).

[15] J. Hall (private communication) CDMS experiment.

[16] M. J. Strassler and K. M. Zurek, Phys. Lett. B 651, 374 (2007).

[17] D. E. Kaplan, M. Luty, and K. Zurek, arXiv:0901.4117.

[18] M. Pospelov, A. Ritz, and M. B. Voloshin, Phys. Lett. B 662, 53 (2008); M. Pospelov and A. Ritz, Phys. Lett. B 671, 391 (2009); M. Pospelov, arXiv:0811.1030.

[19] D. Hooper and K. M. Zurek, Phys. Rev. D 77, 087302 (2008).

[20] J. Hisano, S. Matsumoto, M. M. Nojiri, and O. Saito, Phys. Rev. D 71, 063528 (2005).

[21] M. Fairbairn and J. Zupan, arXiv:0810.4147.

[22] J. Hisano, M. Kawasaki, K. Kohri, T. Moroi, and K. Nakayama, Phys. Rev. D 79, 083522 (2009).

[23] T. Hur, H. S. Lee, and C. Luhn, J. High Energy Phys. 01 (2009) 081; T. Hur, H. S. Lee, and S. Nasri, Phys. Rev. D 77, 015008 (2008); H.S. Lee, Phys. Lett. B 663, 255 (2008).

[24] M. J. Strassler, arXiv:hep-ph/0607160.

[25] J.L. Feng and J. Kumar, Phys. Rev. Lett. 101, 231301 (2008).

[26] N. Arkani-Hamed and N. Weiner, J. High Energy Phys. 12 (2008) 104.

[27] P. J. Fox and E. Poppitz, Phys. Rev. D 79, 083528 (2009).

[28] Y. Nomura and J. Thaler, Phys. Rev. D 79, 075008 (2009).

[29] B. Holdom, Phys. Lett. 166B, 196 (1986). 